\title{
Thermal expansion of the Ag(111) surface measured by x-ray scattering
}

\author{
C. E. Botez, W. C. Elliott, and P. F. Miceli \\ Department of Physics and Astronomy, University of Missouri-Columbia, Columbia, Missouri 65211 \\ P. W. Stephens \\ Department of Physics, State University of New York, Stony Brook, New York, 11794
}

(Received 29 September 2000; published 1 March 2001)

\begin{abstract}
We have investigated the structure of the $\mathrm{Ag}(111)$ surface, for temperatures between 300 and $1100 \mathrm{~K}$ (90\% of the bulk melting point), using synchrotron x-ray diffraction. Our data show no evidence of the anomalously large surface thermal expansion previously reported by medium-energy ion-scattering [Phys. Rev. Lett. 72, 3574 (1994)]. At all temperatures we find that the interlayer separations at the surface differ from their bulk counterparts by less than $1 \%$, indicating that the surface expands similarly to the underlying bulk crystal. This behavior is in good agreement with results from molecular dynamics simulations.
\end{abstract}

DOI: 10.1103/PhysRevB.63.113404

PACS number(s): 68.35.Ja, 61.10.Kw, 68.35.Bs

As the temperature of a metal is raised toward its bulk melting point, the separations between the atomic layers at the surface can expand considerably faster than the ones in the bulk. This behavior, first observed on $\mathrm{Pb}(110),{ }^{1}$ is believed to originate from an enhanced anharmonicity of the surface vibrations, whose other manifestations include roughening and surface premelting. ${ }^{2}$ Subsequent experiments have also revealed an enhanced surface thermal expansion, although somewhat smaller in magnitude, for a number of other low Miller-index metallic surfaces. ${ }^{3-5}$ However, extensive efforts to quantitatively explain these experimental results have not led to a consensus on a correct theoretical description. ${ }^{6-10}$

Recently, a medium-energy ion-scattering (MEIS) study of $\mathrm{Ag}(111)^{3}$ reported an unexpectedly large high-temperature surface thermal expansion, where the relaxation of the first interlayer spacing, $\Delta_{12}=\left[\left(d_{12}-d\right) / d\right]$, was observed to increase from $-2.5 \%$, its value at temperatures between 300 and $670 \mathrm{~K}$, to $+10 \%$ at $T=1100 \mathrm{~K}$. Here, $d_{12}$ is the separation between the first and the second atomic layers at the surface and $d$ is the interlayer spacing in the bulk crystal. Such dramatic structural changes, unanticipated for the close-packed (111) surface, ${ }^{11}$ have attracted considerable theoretical interest. Molecular dynamics (MD) simulations ${ }^{7,8}$ and quasiharmonic approximation (QHA) calculations ${ }^{9,10}$ have been used to investigate the thermal expansion of $\mathrm{Ag}(111)$ but, surprisingly, the two methods yielded substantially different results. While the former (MD) indicates that the surface expands almost bulk-like at all temperatures between $200 \mathrm{~K}$ and $1200 \mathrm{~K}$, in apparent contradiction to the MEIS experiment, the latter (QHA) predicts a large hightemperature surface thermal expansion, somewhat resembling the experimental findings. However, neither the magnitude nor the temperature dependence of the top interlayer relaxation observed in the MEIS experiment was accurately described by the QHA studies. Furthermore, it has been suggested $^{12}$ that the QHA method overestimates the surface thermal expansion, particularly at high temperatures.

In order to clarify the thermal expansion of $\operatorname{Ag}(111)$, we have studied this surface for temperatures between 300 and $1100 \mathrm{~K}$ using $\mathrm{X}$-ray reflectivity, which is a technique that is well known for its ability to accurately determine the surface and bulk interlayer spacings simultaneously. Our data show no evidence of a large surface thermal expansion. At all temperatures we find that the relaxation $\Delta_{12}$ is less than $1 \%$, indicating that the surface and the bulk expand similarly. We also observe that the mean-square amplitude of the surfacenormal vibrations for the atoms in the top layer, $\left\langle u_{1}^{2}\right\rangle$, increases with temperature faster than its bulk counterpart, $\left\langle u_{\text {bulk }}^{2}\right\rangle$, so that at $T=1080 \mathrm{~K}$, the surface-to-bulk ratio of the vibration amplitudes $\left\langle u_{1}^{2}\right\rangle /\left\langle u_{\text {bulk }}^{2}\right\rangle$ is about $30 \%$ larger than at room temperature. These experimental results are in good agreement with the MD simulations.

Our experiment was performed at the National Synchrotron Light Source (NSLS) on the SUNY X3B2 beam line. The $2 \times 12 \mathrm{~mm}$ diam. Ag sample was mechanically polished so that the surface-normal was parallel to the [111] direction to within $0.1^{\circ}$. Further surface preparation was achieved in an ultrahigh vacuum (UHV) chamber (base pressure less than $10^{-10}$ Torr), by repeated cycles of $\mathrm{Ar}^{+}$sputtering and $1000 \mathrm{~K}$ annealing. Electron bombardment was used to heat the sample while the temperature was monitored and stabilized $( \pm 1 \mathrm{~K})$ by a temperature controller. Before collecting each set of data the temperature was accurately determined by direct measurement of the lattice constant and the cleanliness of the surface was verified by Auger-electron spectroscopy. No contaminants were detected at any of the temperatures used in this study. X-ray scattering data were collected by scanning across the specular rod, for an extended range of values of the perpendicular momentum transfer, $Q_{z}$, at temperatures between 300 and $1100 \mathrm{~K}$. The specular reflectivity ${ }^{13}$ was obtained from the integrated intensity measured at each $Q_{z}$ and the transverse scans permitted the background, which includes thermal diffuse scattering, to be determined and subtracted. The angular full-width at halfmaximum (FWHM) of the transverse profiles was observed to decrease with increasing $Q_{z}$. As described in Ref. 14, this $Q_{z}$-dependence can be used to determine the diameter of the average-sized facet on the surface (in-plane correlation length), $L$, and the mosaic angular spread, $\Delta \omega_{0}$. At room temperature we obtain $L=8000 \AA$ and $\Delta \omega_{0}=0.038^{\circ}$. 
$\mathrm{X}$-ray scattering is well established as a technique that can reveal the details of surface structure on an atomic-scale. ${ }^{13}$ The weak interaction of $\mathrm{x}$-rays with condensed matter simplifies the interpretation of the scattering data and permits the surface to be measured simultaneously with the underlying bulk crystal structure. X-rays have been used extensively in studies of metal surfaces to investigate surface crystallography, ${ }^{15}$ surface morphology ${ }^{16,17}$ and surface thermal expansion., ${ }^{4,18}$

Our experimental data are analyzed according to a kinematic scattering model, where the specular reflectivity is expressed as a sum over the positions of each atomic layer:

$$
\begin{aligned}
& R\left(Q_{z}\right) \\
& \quad=c P \frac{F\left(Q_{z}\right)}{Q_{z}^{2}}\left|f\left(Q_{z}\right)\right|^{2}\left|V\left(Q_{z}\right)\right|^{2} \mid \sum_{n=1}^{\infty} e^{-\left.\frac{1}{2}\left\langle u_{n}^{2}\right\rangle Q_{z}^{2} e^{-i z_{n} Q_{z}}\right|^{2} .}
\end{aligned}
$$

Here, $Q_{z}$ is the momentum transfer measured perpendicular to the surface, $c$ is a constant scaled to the experimental data, since the reflectivity was not measured in absolute units, $P$ is the polarization factor ( $=1$ in our experiment), $F\left(Q_{z}\right)$ is the geometrical correction for the beam footprint that gives the number of x-rays striking the surface, and $f\left(Q_{z}\right)$ is the atomic form factor. $V\left(Q_{z}\right)=\left\langle e^{i Q_{z} h}\right\rangle$ accounts for surface roughness, where $h$ is the laterally varying surface height and \langle\rangle is a lateral average. However, we have previously demonstrated $^{14,17}$ that $\mathrm{Ag}(111)$ surfaces prepared according the method used here are virtually flat, consistently having a rms roughness less than $0.5 \AA$. This has a negligible impact on the reflectivity and therefore we use $V=1$ in the analysis.

With most quantities in Eq. (1) established, we are left only with the factors relating to the surface relaxation: $z_{n}$, the vertical coordinate of the $n$th layer beneath the surface and $\left\langle u_{n}^{2}\right\rangle$, the mean-square amplitude of the surface-normal atomic vibrations of layer $n$. In the bulk of the crystal, all of the atomic layers are equidistant, $z_{n}=-(n-1) d$, and have the same vibrational properties $\left\langle u_{n}^{2}\right\rangle=\left\langle u_{\text {bulk }}^{2}\right\rangle$. Near the surface, however, the first few atomic layers relax inward or outward with respect to their bulk-terminated positions due to modified restoring forces that arise from the loss of nearest neighbors; thus, the interlayer separations at the surface, $d_{n, n+1}=z_{n}-z_{n+1}$, are different from the bulk value. Also, the surface-normal vibration amplitudes of the atoms in the top layers are larger than in the bulk, especially at high temperatures. Depending on their magnitude, these deviations from a perfectly truncated lattice can cause measurable changes of the reflectivity curve from its ideal-surface line shape.

The sensitivity of the technique is illustrated in Fig. 1, which shows the effect of an expanded or contracted top interlayer on the $Q_{z}$ dependence of the specular reflectivity calculated according to Eq. (1). The reflectivity from an unrelaxed $\operatorname{Ag}(111)$ surface $\left(\Delta_{12}=0\right)$ is given by the solid curve, whereas the dashed and the dash-dotted curves correspond to an expanded $\left(\Delta_{12}=+4 \%\right)$ and contracted $\left(\Delta_{12}=\right.$ $-4 \%)$ surface, respectively. In all the cases the reflectivity is calculated for room temperature, assuming that the vibra-

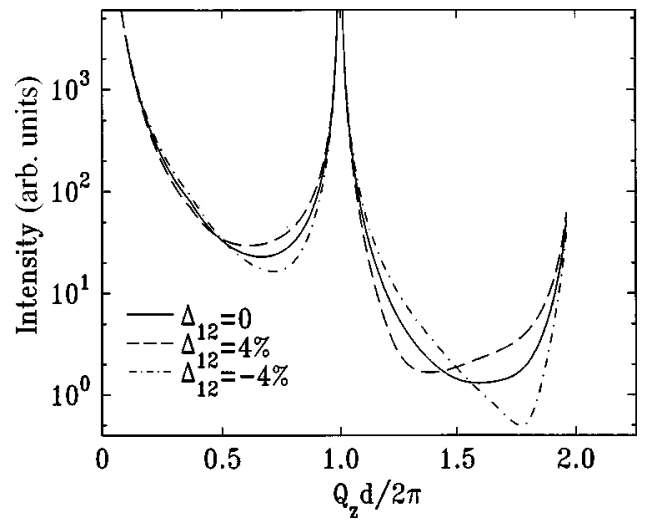

FIG. 1. The specular reflectivity, calculated for $\operatorname{Ag}(111)$ at room temperature, demonstrates the sensitivity of the method to top interlayer expansion and contraction. The solid curve corresponds to a bulk terminated surface, whereas the dashed and the dash-dotted curves correspond to a top interlayer that is $4 \%$ expanded and contracted relative to the bulk crystal, respectively.

tion amplitudes at the surface are the same as in the bulk. The differences among the three line shapes are substantial, particularly at higher $Q_{z}$ values.

Our experimental results are presented in Fig. 2, which shows the specular reflectivity from $\mathrm{Ag}(111)$ for an extended

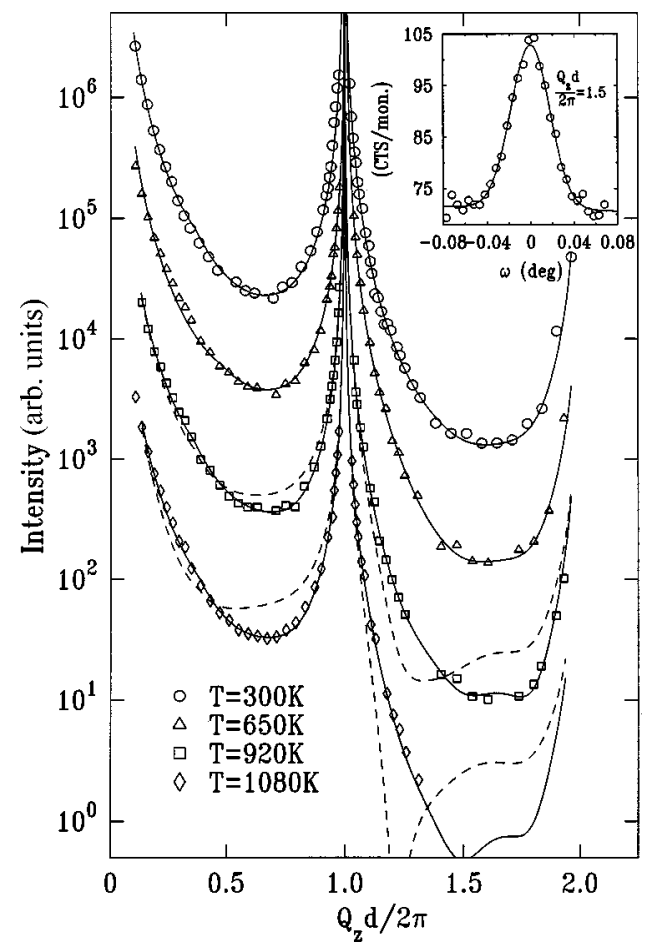

FIG. 2. The measured (open symbols) specular reflectivity of $\mathrm{Ag}(111)$ is shown at different temperatures. Least-square fits of Eq. (1) to the data are shown by the solid curves, whereas the dashed curves are calculated for the large surface expansion reported in Ref. 3: $\Delta_{12}=5 \%$ at $T=920$ and $\Delta_{12}=10 \%$ at $T=1080 \mathrm{~K}$, while keeping the other parameters at their best-fit values. As can be seen, the large surface expansion does not reproduce the x-ray data. The inset shows a transverse scan (open circles) taken at the out-ofphase $(3 / 2,3 / 2,3 / 2)$ position, where the curve is a guide to the eye. 
range of $Q_{z}$, at four different temperatures. The data (open symbols) represent the integrated intensity obtained from transverse scans through the specular rod and a typical scan, taken at the $(3 / 2,3 / 2,3 / 2)$ anti-Bragg position at $T=300 \mathrm{~K}$, is shown in the inset. Due to the combined effects of strong Debye-Waller attenuation of the specular rod and large thermal diffuse scattering, a slightly reduced $Q_{z}$ range was measured at the highest temperature. The solid curves represent best fits of Eq. (1) to the data and were obtained from a nonlinear least-squares analysis. Allowed to vary were the top two interlayer relaxations, $\Delta_{12}$ and $\Delta_{23}$ [where $z_{1}$ $=d\left(\Delta_{12}+\Delta_{23}\right), \quad z_{2}=d\left(\Delta_{23}-1\right)$ and $\left.z_{3}=-2 d\right]$, and the mean-square amplitudes of the surface-normal vibrations for the atoms in the top three layers, $\left\langle u_{1}^{2}\right\rangle,\left\langle u_{2}^{2}\right\rangle$, and $\left\langle u_{3}^{2}\right\rangle$. The structural and vibrational parameters in the remaining layers were kept at their bulk values $\left[z_{n}=-(n-1) d\right.$ and $\left\langle u_{n}^{2}\right\rangle$ $\left.=\left\langle u_{\text {bulk }}^{2}\right\rangle\right]$. For each temperature the vibration amplitudes in the bulk were calculated using the Debye model. ${ }^{19}$

Several notable features are displayed by the data. First, there is a remarkable resemblance between the reflectivity profile measured at $300 \mathrm{~K}$ and the ideal reflectivity of Fig. 1 (solid curve), suggesting that the room temperature surface structure is not very different from the perfect bulktermination. Furthermore, at higher temperatures $(650,920$, and $1080 \mathrm{~K}$ ) the measured reflectivity profiles do not display the asymmetries characteristic of large surface layer relaxation, remaining qualitatively similar to the room temperature data. Therefore, little surface relaxation occurs between 300 and 1080 K. For comparison, we calculated the reflectivity profiles at the higher temperatures using the first interlayer spacings from the MEIS experiment. As can be seen by the dashed curves, those interlayer spacings are not consistent with our experimental data. Finally, at all temperatures, we observe that the $Q_{z}$ dependence of the measured reflectivity shows a strong Debye-Waller attenuation, suggesting that the surface-normal vibration amplitudes in the surface layers are enhanced with respect to the ones in the bulk.

Figure 3 summarizes the results of the data analysis. The temperature dependence of the first interlayer relaxation, $\Delta_{12}$, is shown in Fig. 3(a). At room temperature we find $\Delta_{12}=-0.1 \pm 0.5 \%$, which indicates that, within the margin of error, the separation between the top two atomic layers at the surface is virtually bulk-like. As the temperature is raised, $\Delta_{12}$ increases very slowly, reaching $0.5 \pm 0.6 \%$ at $T$ $=920 \mathrm{~K}$ and $1.05 \pm 1.2 \%$ at $T=1080 \mathrm{~K}$ (the larger error bar at $1080 \mathrm{~K}$ is due to the more limited range over which data were collected at this temperature). An even smaller relaxation resulted for the second interlayer $\left(\left|\Delta_{23}\right| \leqslant 0.4 \%\right.$ at all temperatures). Essentially, our data show that the surface relaxation changes very little over the measured temperature range, which extends to nearly $90 \%$ of the bulk melting point. Figure 3(b) shows the temperature dependence of the mean-square amplitude of the surface-normal vibrations for the atoms in the first and second layers. For comparison, the vibration amplitudes in the bulk, ${ }^{19}\left\langle u_{\text {bulk }}^{2}\right\rangle$, are shown by the filled symbols. At room temperature, we observe that $\left\langle u_{1}^{2}\right\rangle$ and $\left\langle u_{2}^{2}\right\rangle$ are enhanced with respect to their bulk counterpart by a factor of 2 and 1.65, respectively. Upon heating, the

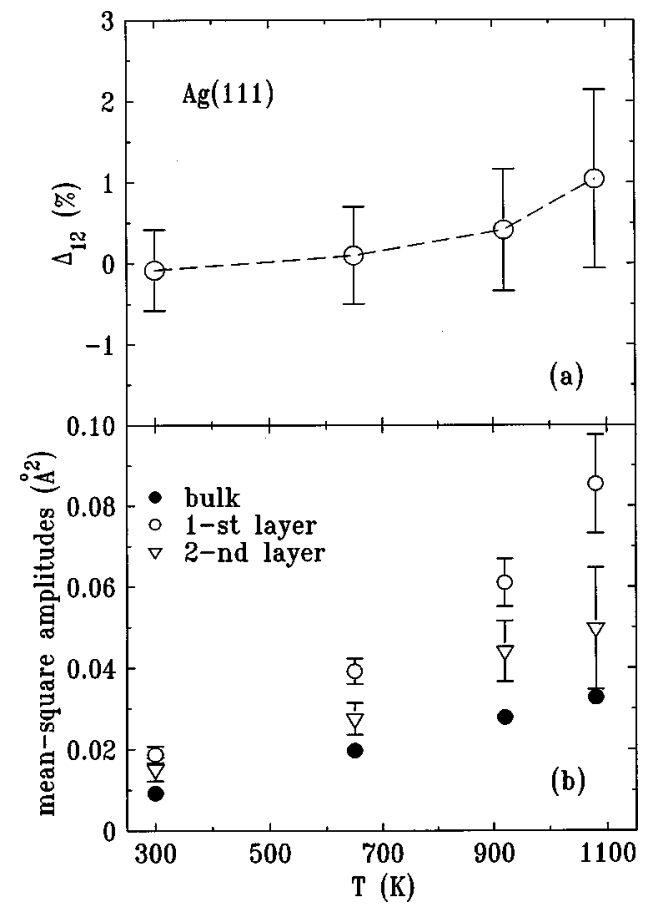

FIG. 3. The temperature dependence of (a) the first interlayer expansion, $\Delta_{12}$, and (b) the mean-square surface-normal vibrational amplitudes obtained from the x-ray scattering experiment are summarized. It is found that $\Delta_{12}$ changes very little with temperature, indicating that the surface expands similarly to the bulk crystal. The mean-square vibrational amplitudes are observed to be larger than in the bulk (solid circles-calculated according to the standard Debye model $^{19}$ ).

vibration amplitudes of the surface atoms increase faster than the ones in the bulk so that, at $T=1080 \mathrm{~K}$, the ratio $\left\langle u_{1}^{2}\right\rangle /\left\langle u_{\text {bulk }}^{2}\right\rangle$ reaches 2.6 , being therefore $30 \%$ larger than at the room temperature. For the atoms in the second layer, $\left\langle u_{2}^{2}\right\rangle /\left\langle u_{\text {bulk }}^{2}\right\rangle$ stays roughly the same at all temperatures. Also, the vibration amplitudes for the atoms in the third layer (not shown here) were found to differ by less than $10 \%$ from the bulk values throughout the measured temperature range.

In Fig. 4, the present x-ray scattering results for the temperature dependence of $\Delta_{12}$ are compared with the findings of the earlier MEIS experiment as well as with two theoretical predictions. Our experimental results (open circles) are consistent with the MD simulations in Ref. 7 (solid circles). Both show a small surface relaxation that changes very little $(<1 \%)$ with temperature throughout the interval between 300 and $1100 \mathrm{~K}$. Recently, more elaborate MD simulations ${ }^{8}$ found essentially the same results. Furthermore, the vibrational amplitudes of the surface atoms obtained by MD simulations are in very good agreement with our experiment. For example, over the full temperature range, the simulations in Ref. 8 give $\left\langle u_{1}^{2}\right\rangle$ ranging from 0.016 to $0.10 \AA^{2}$ whereas our experimental values range from 0.019 to $0.09 \AA^{2}$. Thus the MD approach gives results consistent with our experiment for both the structural and the vibrational properties of $\operatorname{Ag}(111)$ surface.

On the other hand the temperature dependence of the surface relaxation reported in the MEIS experiment ${ }^{3}$ (open 


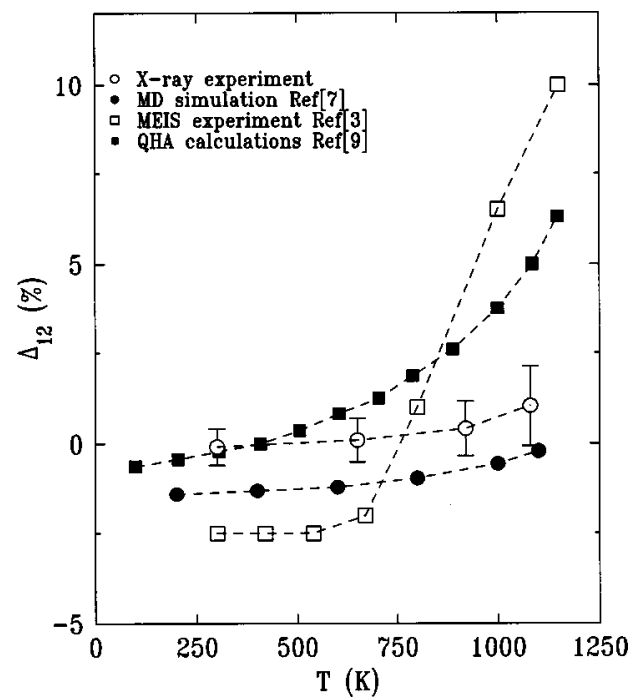

FIG. 4. The temperature dependence of the first interlayer relaxation, $\Delta_{12}$, obtained from our x-ray scattering experiment (open circles) is compared to the MEIS experiment ${ }^{3}$ (open squares), as well as to MD simulations ${ }^{7}$ (solid circles) and QHA calculations ${ }^{9}$ (solid squares). The MD simulations exhibit a small temperature dependence that very closely matches the $\mathrm{x}$-ray scattering results.

squares) exhibits a very different behavior: $\Delta_{12}$ is almost constant up to $670 \mathrm{~K}$, above which, it increases abruptly, reaching $+10 \%$ at $T=1150 \mathrm{~K}$. This feature is not observed in any of the MD or QHA studies. Quasiharmonic approximation calculations, ${ }^{9}$ using the complete phonon spectrum, yield a gradual increase of $\Delta_{12}$ with temperature (solid squares) that is not consistent with either experiment. Although the QHA calculations permit a large hightemperature expansion $\left(\Delta_{12}=6.3 \%\right)$, its value is significantly smaller than the one observed in the MEIS experiment $\left(\Delta_{12}=10 \%\right)$.

In summary, our x-ray scattering experiment shows that the $\operatorname{Ag}(111)$ surface relaxation remains small $\left(\left|\Delta_{12}\right| \leqslant 1 \%\right)$, varying little with temperature throughout the interval between 300 and $1100 \mathrm{~K}$. This indicates that, contrary to previous MEIS results, the surface and the bulk expand similarly at all temperatures up to $90 \%$ of the bulk melting point. Our measured surface thermal expansion and mean-square vibrational amplitudes confirm the results from molecular dynamics simulations that point toward a rather small enhancement of the anharmonic effects at the $\operatorname{Ag}(111)$ surface.

Support is acknowledged from the National Science Foundation under Contract Nos. (PWS) DMR-9202528 and (PFM, CEB, WCE) DMR-9623827 and the Midwest Superconductivity Consortium (MISCON) under DOE Grant No. DE-FG02-90ER45427. The SUNY X3 beam line is supported by the DOE, under Contract No. DE-FG0286ER45231, and the NSLS was supported by the DOE, Division of Material Sciences and Division of Chemical Sciences.
${ }^{1}$ J. W. F. Frenken, F. Hussen, and J. F. van der Veen, Phys. Rev. Lett. 58, 401 (1987)

${ }^{2}$ H.-N. Yang, T.-M. Lu, and G.-C. Wang, Phys. Rev. Lett. 63, 1621 (1989); Y. Cao and E. H. Conrad, ibid. 64, 447 (1990); C. S. Jayanthi, E. Tosatti, and L. Pietronero, Phys. Rev. B 31, 3456 (1985).

${ }^{3}$ P. Satirìs, H. C. Lu, and T. Gustafsson, Phys. Rev. Lett. 72, 3574 (1994).

${ }^{4}$ G. Helgesen, D. Gibbs, A. P. Baddorf, D. M. Zehner, and S. G. J. Mochrie, Phys. Rev. B 48, 15320 (1993).

${ }^{5}$ K. Pohl, J.-H. Cho, K. Terakura, M. Scheffler, and E. W. Plummer, Phys. Rev. Lett. 80, 2853 (1998); K. H. Chae, H. C. Lu, and T. Gustafsson, Phys. Rev. B 54, 14082 (1996); Y. Cao and E. Conrad, Phys. Rev. Lett. 65, 2808 (1990).

${ }^{6}$ M. Lazzeri and S. de Gironcoli, Phys. Rev. Lett. 81, 2096 (1998).

${ }^{7}$ L. J. Lewis, Phys. Rev. B 50, 17693 (1994).

${ }^{8}$ A. N. Al-Rawi, A. Kara, and T. S. Rahman, Surf. Sci. 446, 17 (2000).

${ }^{9}$ J. Xie, S. de Gironcoli, S. Baroni, and M. Scheffler, Phys. Rev. B 59, 970 (1999).
${ }^{10}$ A. Kara, P. Staicov, A. N. Al-Rawi, and T. Rahman, Phys. Rev. B 38, R13 440 (1997); S. Narashimhan and M. Scheffler, Z. Phys. Chem. 202, 253 (1997).

${ }^{11}$ H. Hakkinen and M. Mannnien, Phys. Rev. B 46, 1725 (1992).

${ }^{12}$ A. N. Al-Rawi, A. Kara, P. Staikov, C. Ghosh, and T. S. Rahman, Phys. Rev. Lett. (to be published).

${ }^{13}$ I. K. Robinson and D. J. Tweet, Rep. Prog. Phys. 55, 599 (1992).

${ }^{14}$ W. C. Elliott, P. F. Miceli, T. Tse, and P. W. Stephens, Physica B 221, 65 (1996).

${ }^{15}$ R. Feidenhans'1, Surf. Sci. Rep. 10, 105 (1989).

${ }^{16}$ I. K. Robinson, E. Vlieg, H. Hornis, and E. H. Conrad, Phys. Rev. Lett. 67, 1890 (1991); E. Vlieg, A. W. Denier van der Gon, J. F. van der Veen, J. E. Macdonald, and C. Norris, ibid. 61, 2241 (1988).

${ }^{17}$ W. C. Elliott, P. F. Miceli, T. Tse, and P. W. Stephens, Phys. Rev. B 54, 17938 (1996).

${ }^{18}$ D. Gibbs, B. M. Ocko, D. M. Zehner, and S. G. J. Mochrie, Phys. Rev. B 38, 7303 (1988).

${ }^{19}$ B. D. Cullity, Elements of X-Ray Diffraction (Addison-Wesley, Reading, 1956). 